\title{
Cardiac Arrest Induces Ischemic Long-Term Potentiation of Hippocampal CA1 Neurons That Occludes Physiological Long-Term Potentiation
}

\author{
James E. Orfila, ${ }^{1}$ Nicole McKinnon, ${ }^{2}$ Myriam Moreno, ${ }^{1}$ Guiying Deng, ${ }^{3}$ Nicholas Chalmers, ${ }^{1}$ \\ Robert M. Dietz, ${ }^{2}$ Paco S. Herson, ${ }^{1,3}$ and Nidia Quillinan $\mathbb{1}^{1}$ \\ ${ }^{1}$ Neuronal Injury Program, Department of Anesthesiology, University of Colorado, Anschutz Medical Campus, Aurora, \\ CO 80045, USA \\ ${ }^{2}$ Department of Pediatrics, University of Colorado, Anschutz Medical Campus, Aurora, CO 80045, USA \\ ${ }^{3}$ Department of Pharmacology, University of Colorado, Anschutz Medical Campus, Aurora, CO 80045, USA
}

Correspondence should be addressed to Nidia Quillinan; nidia.quillinan@ucdenver.edu

Received 27 November 2017; Revised 15 March 2018; Accepted 5 April 2018; Published 26 April 2018

Academic Editor: Paola Bonsi

Copyright (C) 2018 James E. Orfila et al. This is an open access article distributed under the Creative Commons Attribution License, which permits unrestricted use, distribution, and reproduction in any medium, provided the original work is properly cited.

\begin{abstract}
Ischemic long-term potentiation (iLTP) is a form of synaptic plasticity that occurs in acute brain slices following oxygen-glucose deprivation. In vitro, iLTP can occlude physiological LTP (pLTP) through saturation of plasticity mechanisms. We used our murine cardiac arrest and cardiopulmonary resuscitation (CA/CPR) model to produce global brain ischemia and assess whether iLTP is induced in vivo, contributing to the functionally relevant impairment of pLTP. Adult male mice were subjected to CA/ $\mathrm{CPR}$, and slice electrophysiology was performed in the hippocampal CA1 region 7 or 30 days later. We observed increased miniature excitatory postsynaptic current amplitudes, suggesting a potentiation of postsynaptic AMPA receptor function after CA/CPR. We also observed increased phosphorylated GluR1 in the postsynaptic density of hippocampi after CA/CPR. These data support the in vivo induction of ischemia-induced plasticity. Application of a low-frequency stimulus (LFS) to CA1 inputs reduced excitatory postsynaptic potentials in slices from mice subjected to CA/CPR, while having no effects in sham controls. These results are consistent with a reversal, or depotentiation, of iLTP. Further, depotentiation with LFS partially restored induction of pLTP with theta burst stimulation. These data provide evidence for iLTP following in vivo ischemia, which occludes pLTP and likely contributes to network disruptions that underlie memory impairments.
\end{abstract}

\section{Introduction}

Ischemic long-term potentiation (iLTP) is an increase in excitatory synaptic strength that occurs immediately following oxygen and glucose deprivation (OGD) in acute brain slices [1-6]. Elevations in extracellular glutamate during OGD cause prolonged activation of postsynaptic $\alpha$-amino3-hydroxy-5-methyl-4-isoxazolepropionic acid (AMPA) and $\mathrm{N}$-methyl-D-aspartic acid (NMDA) receptors, resulting in an influx of sodium and calcium. Rises in intracellular calcium stimulate calcium/calmodulin-dependent protein kinase (CAMKII) signaling, which potentiates postsynaptic excitatory function via increased AMPA receptor phosphorylation and expression at the synapse. There is some indirect evidence to support that iLTP occurs following in vivo ischemia. Previously, we demonstrated increased activation of CAMKII within hours of global ischemia induced by cardiac arrest [7]. There is also evidence to support acute activation of CAMKII and increased NMDA receptor expression in the hippocampus within hours of in vivo focal ischemia [8]. However, it is unknown whether acute activation of CAMKII seen following in vivo ischemia causes synaptic potentiation in the hippocampus or whether ischemic LTP is maintained for days beyond the ischemic event.

Shared mechanisms between ischemic and physiologic LTP suggest that it is likely these plasticity processes would occlude one another, as has been described in studies where acute hippocampal brain slices were subjected to in vitro 
ischemia $[3,4]$. Physiological hippocampal long-term potentiation (pLTP) is an experience- or frequency-dependent increase in synaptic strength and is a cellular substrate for learning and memory. Similar to iLTP, pLTP occurs through an NMDA and CAMKII-dependent increase in synaptic AMPA receptor function [9-12]. Memory deficits in cardiac arrest survivors are attributed to ischemic injury to the hippocampus that causes loss of pyramidal CA1 neurons $[13,14]$. In addition to neuronal cell death, global ischemia causes persistent deficits in pLTP in surviving neurons of the CA1 [7, 15-19]. Therefore, pLTP deficits caused by brain ischemia likely contribute to memory deficits, and therapies that restore pLTP have the potential to improve cognitive function after CA/CPR. Acute neuroprotective interventions that reduce CA1 injury can also prevent pLTP deficits; however, there is no strategy that targets LTP deficits at delayed time points and that is independent of preventing neuronal cell death $[7,15,19,20]$. The goal of this study was to determine whether in vivo global ischemia from cardiac arrest causes ischemic LTP that prevents physiological LTP.

\section{Methods}

2.1. Experimental Animals and Cardiac Arrest Model. The Institutional Animal Care and Use Committee (IACUC) at the University of Colorado approved all experimental protocols in accordance with the National Institutes of Health and guidelines for the care and use of animals in research. Analysis was performed with investigators blinded to experimental groups. Adult (8-12-week-old) male C57B16 (Charles River, Wilmington, MA) mice were subjected to CA/CPR as previously described during the $\mathrm{ON}$ light cycle [21-23]. A total of 54 animals were included in this study.

Briefly, anesthesia was induced with $3 \%$ isoflurane and maintained with $1.5-2 \%$ isoflurane in oxygen-enriched air using a nose cone. Temperature probes were inserted in the left ear and rectum to monitor tympanic (head) and body temperature simultaneously. A PE-10 catheter was inserted into the right internal jugular vein for drug administration. Needle electrodes were placed subcutaneously on the chest for continuous electrocardiogram (EKG) monitoring. Animals were endotracheally intubated and connected to a mouse ventilator (MiniVent Ventilator, Harvard Apparatus). Cardiac arrest was induced with injection of $50 \mu \mathrm{l} \mathrm{KCl}$ $(0.5 \mathrm{M})$ via the jugular catheter and confirmed by asystole on EKG. During cardiac arrest, the endotracheal tube was disconnected, anesthesia stopped, and body temperature was allowed to spontaneously decrease to a minimum of $35.5^{\circ} \mathrm{C}$, and head temperature was maintained at $37.5^{\circ} \mathrm{C}$. Resuscitation began eight minutes after induction of cardiac arrest by slow injection of $0.5-1.0 \mathrm{ml}$ epinephrine solution (16 $\mu \mathrm{g}$ epinephrine/ml $0.9 \%$ saline), chest compressions, and ventilation with $100 \%$ oxygen at a respiratory rate of 200 breaths/min. Chest compressions were stopped as soon as spontaneous circulation was restored. Resuscitation was abandoned if spontaneous circulation was not restored within 2.5 minutes. Mice were extubated after they recovered an adequate respiratory rate and effort. Sham controls underwent the same procedures as mice undergoing cardiac arrest including anesthesia, intubation, placement of the jugular catheter, EKG leads, and temperature management. Sham controls did not receive $\mathrm{KCl}$ or epinephrine injections or chest compressions. The animals were placed in a singlehoused static recovery cage on a heated water blanket $\left(35^{\circ} \mathrm{C}\right)$ for the first 24 hours of recovery and at ambient room temperature for long-term recovery (up to 30 days). Mice received soft food and subcutaneous saline for 3 days after surgery and had free access to water and regular chow.

2.2. Acute Slice Preparation. Following CA/CPR or sham surgery, mice were anesthetized with isoflurane $(3.5 \%)$ and transcardially perfused with ice-cold artificial cerebral spinal fluid (ACSF) containing (in mmol/l) $126 \mathrm{NaCl}, 2.5 \mathrm{KCl}, 2.5$ $\mathrm{CaCl}_{2}, 1.2 \mathrm{MgCl}_{2}, 1.2 \mathrm{NaH}_{2} \mathrm{PO}_{4}, 21.4 \mathrm{NaHCO}_{3}$, and $11 \mathrm{D}-$ glucose, bubbled with $95 \% \mathrm{O}_{2} / 5 \% \mathrm{CO}_{2}$ to maintain $\mathrm{pH}$ of 7.4. Mice were decapitated and brains were rapidly removed. Horizontal hippocampal sections $(300 \mu \mathrm{M})$ were cut in icecold ACSF using a VT1200S Vibratome (Leica, Buffalo Grove, IL, USA) and then maintained at room temperature for at least 30 minutes prior to recording.

2.3. Miniature Excitatory Postsynaptic Currents (mEPSCs). Whole-cell recordings were performed at room temperature $\left(22^{\circ} \mathrm{C}\right)$ in a submersion chamber and were continuously perfused with ACSF containing picrotoxin (PTX, $100 \mu \mathrm{M}$ ) and tetrodotoxin (TTX, $250 \mathrm{nM}$ ). Recordings were obtained using borosilicate glass pipettes that were fabricated using a Flaming/Brown heat puller (Sutter Instruments, Novato, $\mathrm{CA}, \mathrm{USA}$ ) to a resistance of 2-4 M $\Omega$. Internal recording solution contained (in mmol/l) $120 \mathrm{~K}$-gluconate, $9 \mathrm{KCl}, 10 \mathrm{KOH}$, $4 \mathrm{NaCl}, 10$ HEPES, 0.05 EGTA, $1 \mathrm{MgCl}_{2}, 4 \mathrm{Na}_{2} \mathrm{ATP}$, and 0.4 $\mathrm{Na}_{2} \mathrm{GTP}$. Series resistance was $<20 \mathrm{M} \Omega$ and did not change more than $20 \%$ during the experiment. Whole-cell voltageclamp recordings were performed at a holding potential of $-70 \mathrm{mV}$. Gap-free continuous recordings were acquired in 3-minute sweeps. Miniature events were identified using Clampfit software with template event detection, and mEPSC amplitude and frequency were quantified for each cell. To generate cumulative probability histograms events from all sham or CA/CPR, mice were pooled.

2.4. Extracellular Field Recording. For extracellular recordings, slices were transferred to an interface recording chamber that was continuously perfused with ACSF $(1.5 \mathrm{ml} / \mathrm{min})$ and warmed to $32^{\circ} \mathrm{C}$. Extracellular field excitatory postsynaptic potentials (fEPSPs) recorded in the stratum radiatum were evoked with a bipolar stimulus electrode positioned in the stratum molecularae/luminaris to evoke glutamate release from Schaffer collaterals $(0.05 \mathrm{~Hz})$. Input-output curves were generated by increasing stimulus intensity in $10 \mu \mathrm{A}$ increments and recording fEPSP slopes. Stimulus intensity was adjusted to produce a fEPSP with a slope that was $50 \%$ of the maximum. A stable baseline fEPSP was recorded for 20 minutes before theta burst stimulation (TBS; 10 trains of $4-100 \mathrm{~Hz}$ pulses) was applied to Schaffer collaterals. fEPSPs were recorded for 60 minutes following TBS, and percent change from baseline was calculated for 
the last 10 minutes of the recording. Low-frequency stimulation (LFS) was delivered for 10 minutes (900 pulses at $0.5 \mathrm{~Hz}$ ), and percent change from baseline was analyzed 20 minutes after LFS. Data were compressed to 1-minute averages, and the extent of LTP or depotentiation was measured as percentage of the baseline fEPSP slope during the last 10 minutes of the recording.

2.5. Western Blot Analysis. Following CA/CPR or sham surgery, mice were deeply anesthetized with isoflurane $(3.5 \%)$, heads were decapitated and brains were rapidly removed. Hippocampi were isolated and rapidly frozen with 2methylbutane on dry ice. Individual hippocampi were homogenized in sucrose buffer containing protease and phosphatase inhibitors using a PTFE tissue grinder in a glass tube. Homogenates were centrifuged at $1000 \times \mathrm{g}$ for 10 minutes to remove cellular debris and nuclei. Supernatant was removed and spun at $10,000 \times \mathrm{g}$ for 15 minutes. This supernatant was collected and spun at $100,000 \times \mathrm{g}$ for 60 minutes, yielding a supernatant that contains the cytosolic cellular fraction (S3). The pellet (P2) was resuspended in triton buffer and then centrifuged at $32,000 \times \mathrm{g}$ for 20 minutes, yielding a pellet (P4) that contains the postsynaptic density (PSD) fraction. This pellet was resuspended in N-PER buffer (Thermo Fisher, Waltham, MA) containing protease and phosphatase inhibitors. The PSD protein concentration was quantified using a BCA kit, and samples were diluted in $4 \mathrm{x}$ denaturing sample buffer to a final concentration of $1 \mu \mathrm{g} / \mu \mathrm{l}$. Protein $(20 \mu \mathrm{g})$ was loaded onto a polyacrylamide gel for protein electrophoresis and transferred to a PVDF membrane. Membranes were blocked in Tris-buffered saline with Tween (TBS-T) containing 5\% BSA or milk. Primary antibody incubations were performed overnight at $4^{\circ} \mathrm{C}$ and detected using horseradish peroxidase-conjugated secondary antibodies. Bands were visualized using a maximum sensitivityenhanced chemiluminescence substrate with the ChemiDoc Gel Imaging System (Bio-Rad, Hercules, CA). Multiple antibodies were probed on each membrane by stripping with Restore Plus stripping buffer after chemiluminescent detection. Integrated volume of bands was normalized to betaactin integrated volume for that sample. Normalized protein expression is presented relative to sham controls.

2.6. Statistics. For electrophysiology experiments, $n$ indicates the number of recordings with no more than two recordings for a given experiment from a single animal. Data are presented as mean \pm SEM. Statistical comparisons were made between two groups using Student's $t$-test and multiple groups using one-way analysis of variance (ANOVA) followed by Dunnett's post hoc comparison of groups relative to control. Statistical comparisons were performed using GraphPad Prism 7.0. Differences with a $p$ value of $<0.05$ were considered significant.

\section{Results}

3.1. Increased AMPA Receptor Function following In Vivo Ischemia. The expression of LTP occurs through an increase in AMPA receptor function resulting from phosphorylation and increased synaptic expression. To directly measure postsynaptic AMPA receptor function, we performed whole-cell recording of miniature EPSCs (mEPSCs) in CA1 neurons 7 days after CA/CPR. Delayed neuronal cell death occurs at 2-3 days postinjury; therefore, by 7 days postinjury, cell death processes are complete and electrophysiology can be performed in surviving neurons that exhibit LTP deficits [7, 19, 22, 24]. Miniature excitatory events were isolated using tetrodotoxin (TTX, $250 \mathrm{nM}$ ) and picrotoxin (PTX, $100 \mu \mathrm{M})$ (Figure 1(a)). Mean mEPSC amplitude, kinetics, and frequency were analyzed using Clampfit template event detection. Cumulative frequency distributions of mEPSC amplitudes were generated by pooling events from recordings in sham ( $n=2,729$ events) and CA/CPR $(n=3,213$ events). The cumulative frequency curve was right-shifted in mice after CA/CPR compared to sham controls, with larger maximum amplitudes (110.7pA versus $56.2 \mathrm{pA}$ ) (Figure 1(b)). The shift to larger events was also detected as an increase in the mean mEPSC amplitude from $16.43 \pm 0.94 \quad(n=12) \quad$ to $20.74 \pm 1.1 \quad(n=15 ; p=0.008)$ (Figure 1(c)). Rise and decay kinetics of mEPSCs were not different between shams and controls (Table 1). There were also no changes in the biophysical properties of neurons that would account for the larger amplitude mEPSCs observed after CA/CPR (Table 1). Event frequency was similar in sham $(1.4 \pm 0.4, n=12)$ and cardiac arrest mice $(1.4 \pm 0.3, n=15 ; p=0.95)$ (Figure $1(d)$ ), indicating no change in synapse number. These data suggest there is increased postsynaptic AMPA receptor function at CA1 synapses following CA/CPR.

Synaptic potentiation results from NMDA receptordependent activation of CAMKII and the subsequent increase in AMPA receptor phosphorylation and expression at postsynaptic sites. Previously, we reported an acute increase in CAMKII activity (T286 phosphorylation) in the hippocampus 3-hour post-CA/CPR, suggesting an ischemia-induced increase in CAMKII activation [7]. To determine whether there are changes in glutamate receptor phosphorylation and expression at delayed time points after cardiac arrest, we isolated the hippocampus from shams and 7 days after $\mathrm{CA} / \mathrm{CPR}$, and protein fractions enriched for postsynaptic densities were subjected to Western blot analysis (Figure 2(a)). We observed an increase in levels of phosphorylated AMPA receptors (GluR1 pS831) from $1.01 \pm 0.03$ $(n=7)$ in shams to $1.23 \pm 0.1 \quad(n=6)$ at 7 days postinjury $(p=0.047)$, consistent with an increase in receptor function (Figure 2(b)). GluR1 AMPA receptor expression (sham: $1 \pm 0.16, n=7$; CA/CPR: $1.28 \pm 0.22, n=7$ ) and GluR2/3 expression (sham: $1 \pm 0.2, n=5$; CA/CPR: 1.25 $\pm 0.16, n=4)$ were not different after cardiac arrest $(p=$ 0.312 and $p=0.36$, resp.) (Figures $2(\mathrm{c})$ and $2(\mathrm{~d})$ ). Ischemic LTP caused by in vitro ischemia can increase NMDA expression [8]. We observed a small increase in NMDA receptor (GluN1) from $1 \pm 0.14(n=5)$ to $1.38 \pm 0.21(n=4)$ expression that was not significant $(p=0.1675)$ (Figure $2(e)$ ). Finally, we saw no change in PSD-95 levels after cardiac arrest (sham: $1 \pm 0.1, n=7$; CA/CPR: $1.05 \pm 0.21, n=7 ; p=$ $0.823)$, suggesting no changes in the overall synapse density (Figure 2(f)). These data are consistent with our mEPSC data 


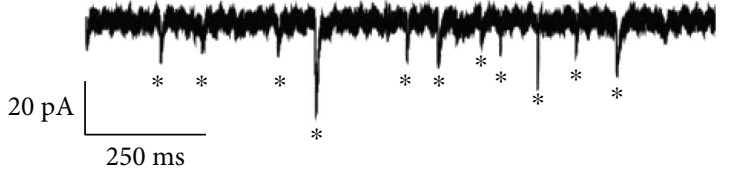

a)



(c)

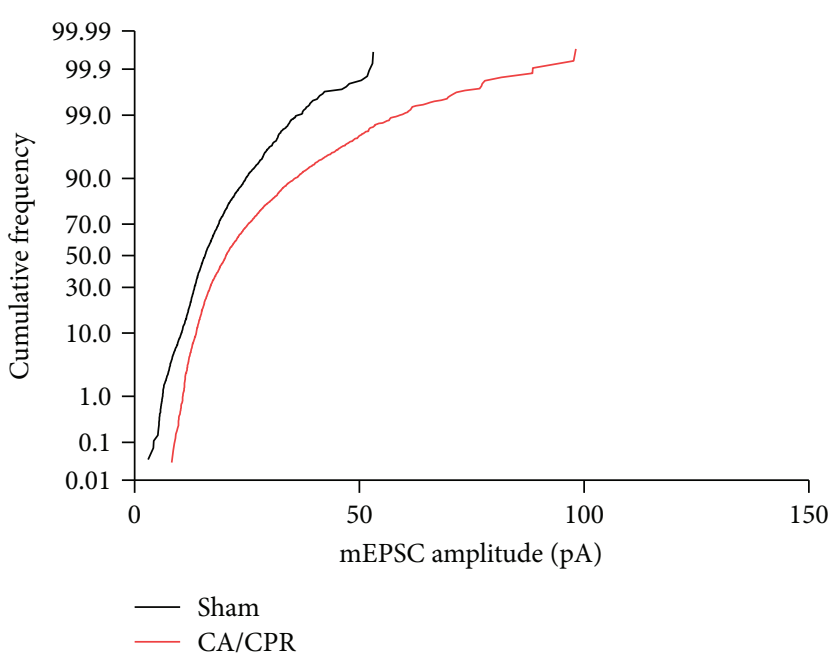

(b)

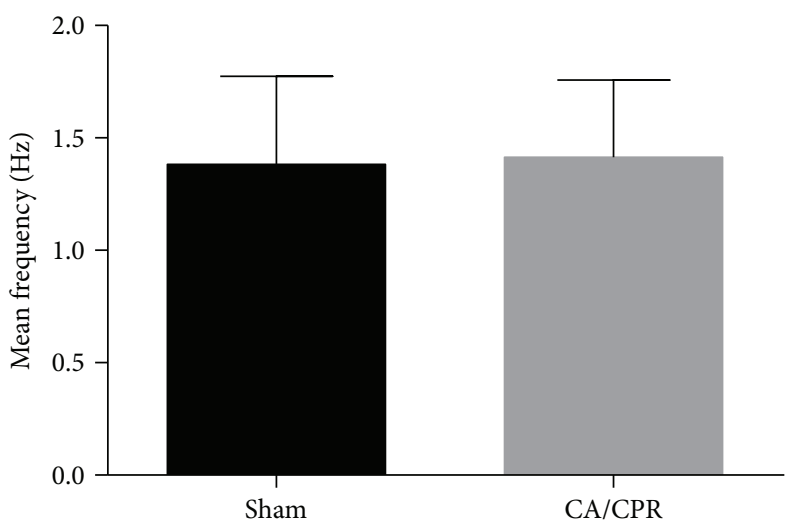

(d)

FIGURE 1: Lasting potentiation of miniature excitatory postsynaptic currents (mEPSCs) induced by cardiac arrest. (a) A representative trace from a sham control of whole-cell voltage clamp recording of mEPSC events recorded from CA1 neurons in acute brain slices. Events were detected with Clampfit software and are indicated with an asterisk. (b) CA/CPR produced a rightward shift in the cumulative frequency distribution of mEPSC amplitudes relative to shams. Events from sham (black, $n=2729$ events) or CA/CPR (red, $n=3213$ events) mice were pooled to generate histograms. (c) CA/CPR produced an increase in mean mEPSC amplitudes compared to sham. Mean mEPSC amplitude was calculated for each recording (sham: $n=12$; CA/CPR: $n=15$ ), and means for groups were compared using Student's unpaired $t$-test $(*$ indicates $p<0.05)$. (d) CA/CPR did not alter synaptic density in CA1 neurons. No change in mean mEPSC frequency was observed between sham and CA/CPR mice. Mean mEPSC frequency was calculated for each recording (sham: $n=12$; CA/CPR: $n=15$ ), and means for groups were compared using Student's unpaired $t$-test.

TABLE 1

\begin{tabular}{lccr}
\hline & Sham & 7 days & 30 days \\
\hline$R_{\mathrm{m}}(\mathrm{MW})$ & $281.0 \pm 30.85(n=10)$ & $230.6 \pm 38.65(n=16)$ & 0.367 \\
$C_{\mathrm{m}}(\mathrm{pF})$ & $5.738 \pm 1.360(n=10)$ & $9.224 \pm 1.845(n=16)$ & 0.1893 \\
PPR (pulse 1/pulse 2) & $1.32 \pm 0.1(n=5)$ & $1.30 \pm 0.08(n=8)$ & 0.889 \\
I/O (slope) & $3.65 \pm 0.59(n=6)$ & $4.57 \pm 0.33(n=6)$ & 0.548 \\
EPSC rise time (ms) & $2.68 \pm 0.27(n=12)$ & $2.37 \pm 0.17(n=15)$ & $3.85 \pm 0.78(n=6)$ \\
EPSC decay time (ms) & $11.60 \pm 1.08(n=12)$ & $12.55 \pm 0.99(n=15)$ & 0.3271 \\
\hline
\end{tabular}

showing increased amplitude and no change in frequency of mEPSC events.

3.2. Depotentiation Restores the Ability to Induce Physiological LTP in Postischemic Neurons. Depotentiation, which is the reversal of LTP, is induced with low-frequency stimulation of synapses that were previously given high-frequency stimulation to induce LTP [25-28]. We hypothesized that ischemic LTP following CA/CPR would be reversed with a depotentiation stimulus. 


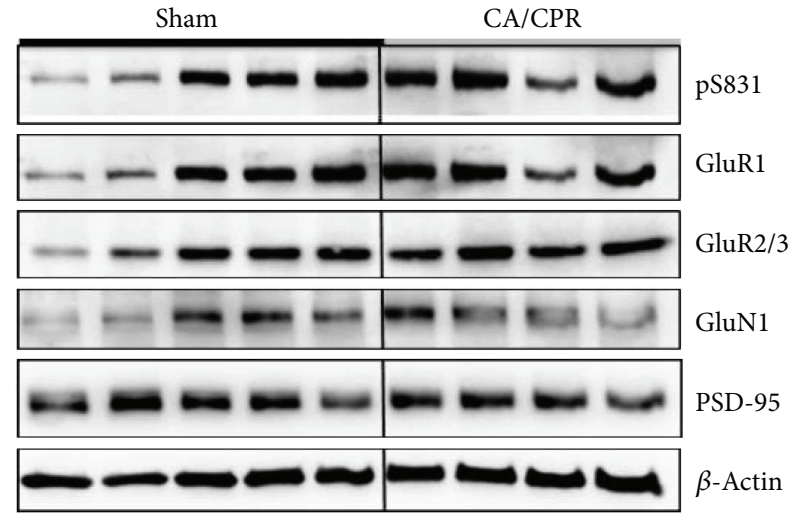

(a)

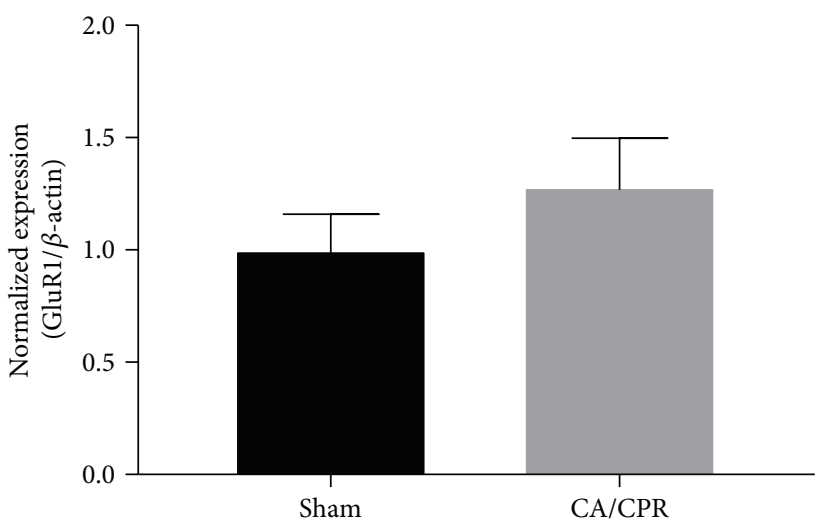

(c)



(e)

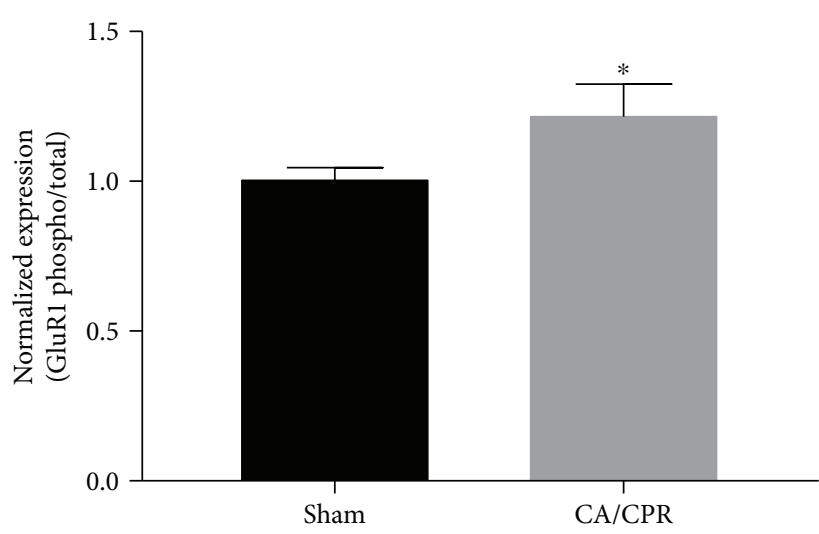

(b)



(d)

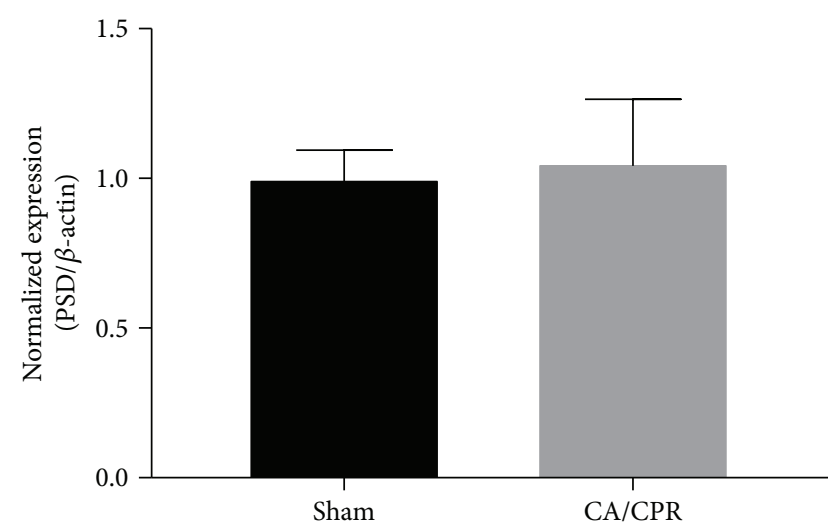

(f)

FIGURE 2: Increased AMPA receptor phosphorylation after CA/CPR. (a) Representative blots of protein expression from synaptic fractions of sham and CA/CPR hippocampus. Blots were cropped to show bands at molecular weight for indicated proteins. (b) Normalized phosphorylated S831: total GluR1 expression was calculated for each sample by dividing optical density of phosphoS831 by total GluR1 density within the same blot. (c) Normalized GluR1 expression was calculated for each sample by dividing optical density of total GluR1 by $\beta$-actin density within the same blot. (d) Normalized GluR2/3 expression was calculated for each sample by dividing optical density of total GluR2/3 by $\beta$-actin density within the same blot. (e) Normalized GluN1 expression was calculated for each sample by dividing optical density of total GluN1 by $\beta$-actin density within the same blot. (f) Normalized PSD-95 expression was calculated for each sample by dividing optical density of PSD-95 by $\beta$-actin density within the same blot. Values were normalized to sham controls. Shams $(n=7)$ and CA/CPR $(n=6)$ groups were compared using Student's $t$-test. $*$ indicates $p<0.05$.

Previous studies use stimulation frequencies ranging between 0.5 and $2 \mathrm{~Hz}$ to depotentiate pLTP without inducing long-term depression (LTD). We found that 900 pulses, delivered at $0.5 \mathrm{~Hz}$, reversed LTP that was induced by a previous theta burst stimulation (TBS) from $182.5 \pm 7.7 \%$ to $132.7 \pm 15.4 \%$ of baseline amplitude $(n=5)$. Importantly, this LFS protocol did not induce LTD in naive controls, having no effect on fEPSP slope 
from baseline following LFS ( $n=6 ; p=0.13$ ) (Figure 3(a)), thus fitting the definition of a depotentiation protocol.

We next tested whether a depotentiation LFS protocol was capable of reducing synaptic strength in mice subjected to $\mathrm{CA} / \mathrm{CPR}$, thus providing further evidence of sustained iLTP. In sham controls, fEPSP slopes were $110 \pm 4.2 \%$ $(n=6)$ of baseline after LFS, an increase that was not statistically significant $(p=0.06)$ (Figure $3(\mathrm{~b}))$. Acute slices prepared 7 days after CA/CPR showed a decrease in fEPSP slope to $83 \pm 10.6 \%(n=7)$ of baseline after LFS, a change that was not statistically different from baseline $(p=0.2)$ but was significantly different than the change observed in controls $(p=0.007)$. At 30 days after CA/CPR, the change in fEPSP slope to $75.73 \pm 7.0 \%(n=5)$ of baseline after LFS was significantly different from baseline $(p=0.015)$ and from the change observed in controls $(p=0.002)$. This provides additional evidence for CA1 synapses being in a potentiated state following in vivo ischemia.

The induction of ischemic LTP by CA/CPR may occlude physiological LTP. To test this, we delivered LFS to induce depotentiation and followed this with TBS in slices from mice at 7 days postinjury. After acquiring a stable 10minute baseline, we delivered LFS, resulting in a decrease of fEPSP slope to $87.7 \pm 0.4 \%(n=5)$ of baseline (Figure $3(\mathrm{c}$ ), dotted line). After 20 minutes, we delivered TBS, which increased fEPSP slope to $115.1 \pm 6.1 \%$ of original baseline, a potentiation of $28 \%(p=0.015)$ (Figure 3(c), shaded blue). These data suggest that LTP mechanisms are saturated, and that reversal of ischemic LTP with LFS partially restores the capacity to induce physiological LTP.

\section{Discussion}

We have provided several pieces of evidence for the presence of sustained ischemic LTP subsequent to in vivo global ischemia caused by cardiac arrest: (1) increased postsynaptic glutamate receptor function, (2) increased postsynaptic glutamate receptor phosphorylation, and (3) the ability to depotentiate CA1 synapses after cardiac arrest. Further, we have shown that ischemic LTP occludes physiological LTP, providing a possible target for interventional strategies to improve memory function after cardiac arrest.

To our knowledge, this is the first study to demonstrate that in vivo ischemia causes synaptic alterations that are consistent with ischemic LTP. Until now, all electrophysiological evidence for this phenomenon comes from in vitro studies using oxygen and glucose deprivation in slices. Therefore, by showing that this phenomenon occurs in vivo, we suggest that this is a mechanism through which memory impairment occurs. Ischemic LTP is similar to physiological LTP in its NMDA receptor dependence, activation of intracellular signaling, and an increase in postsynaptic AMPA receptor function $[3,5]$. The stimulus for inducing ischemic LTP is the massive increase in extracellular glutamate that occurs within minutes of the onset of ischemia [29-33]. Importantly, it is this massive increase in extracellular glutamate that stimulates excitotoxic cell death. By enhancing postsynaptic responses to extracellular glutamate, ischemic LTP likely amplifies excitotoxicity mechanisms [34,35], but it is unclear from in vitro studies what contribution this phenomenon has to CA1 injury after CA/CPR. Similarly, it is difficult to disentangle ischemic LTP and excitotoxicity in vivo, as they have similar induction mechanisms. Previous work from our laboratory and others has shown that pharmacological or genetic interventions reduce NMDA receptor activation or CAMKII activation, not only reducing neuronal cell death but also preserving physiological LTP [7, 19]. It is possible that neuroprotective strategies prevent LTP impairments, in part, by blocking ischemic LTP.

Our strongest evidence for ischemic LTP comes from electrophysiological recordings that demonstrate increased miniature EPSC amplitude. The advantage of this method is that we can specifically assess postsynaptic receptor function in CA1 region of the hippocampus. In our recording conditions, increased mEPSC amplitudes likely represent increased AMPA rather than NMDA receptor function. Other groups have reported that iLTP observed at acute time points is a result of increased expression and function of NMDA receptors $[1,3,8]$. However, we have previously demonstrated no change in NMDA receptor function or expression at 7 days after $\mathrm{CA} / \mathrm{CPR}$, consistent with our results here $[19,20]$. These differences may be due to the use of in vivo versus in vitro models, or that our studies were performed days, rather than hours after the ischemic insult. Analysis of glutamate receptor expression performed here was from the synaptic fraction of the entire hippocampus, not just the CA1 region. Therefore, increases in CA1 receptor expression may be underrepresented within this pool. Regardless, our Western blot data provided evidence for increased phosphorylation of the GluR1 AMPA receptor subunit, which is consistent with our electrophysiological data. There have been mixed results as to whether iLTP has a presynaptic mechanism $[3,6]$. We failed to detect differences in paired-pulse ratio, suggesting a postsynaptic mechanism for iLTP induced by CA/CPR. Others have reported that impaired hippocampal LTP following global ischemia is associated with reduced spine densities [36-38]. However, we did not detect a reduction in mEPSC frequency and PSD95 expression, which are indirect measures of the number of synapses. Therefore, our data is consistent with ischemiainduced changes in plasticity without changes in the number of functional synapses. However, further experiments are needed to rule out an ischemia effect on spine density that may contribute to impaired synaptic plasticity.

Physiological LTP and ischemic LTP have shared mechanisms and, therefore, have the ability to occlude one another. Indeed, tetanic stimulation delivered just prior to OGD prevents ischemic LTP and vice versa [4-6]. Remarkably, we saw that depotentiation prior to theta burst stimulation allowed for the induction of physiological LTP. Therefore, these results support in vitro findings that ischemic LTP saturates plasticity mechanisms to occlude physiological LTP. The stimulus frequency used to depotentiate had no effect on naive control slices, giving us confidence that we induced the depotentiation of synapses, rather than inducing long-term depression, which has different signaling mechanisms. While there was some physiological LTP following depotentiation, LFS did not restore completely back 



(a)

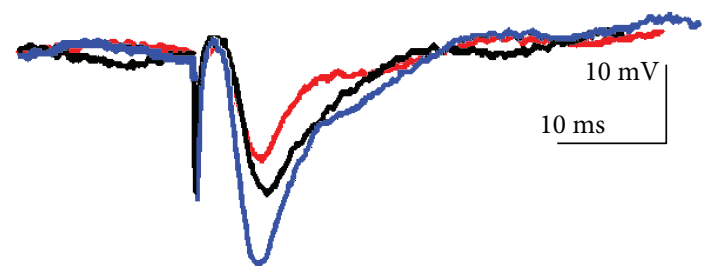
(1) Baseline
(2) LFS
(3) TBS

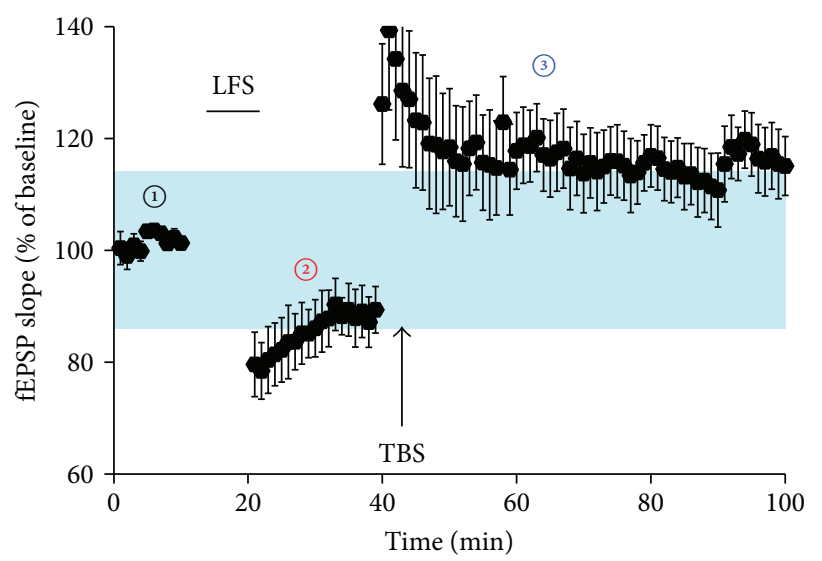

(c)

(d)

FIGURE 3: Depotentiation with low-frequency stimulation (LFS) reversed ischemic LTP and partially restored physiological LTP. (a) LFS depotentiates physiological LTP. 20 minutes after theta burst stimulation (TBS), LFS was delivered for 10 minutes (900 pulses at $0.5 \mathrm{~Hz}$ ), resulting in a significant reduction in fEPSP slope (grey squares). LFS delivered to naive slices that did not receive TBS did not alter fEPSP slope (black circles). (b) LFS was delivered to slices from sham control (black circles) or 7 (blue triangles) or 30 days (red squares) after CA/CPR. LFS reduces fEPSC only in mice that were subjected to CA/CPR, indicating a reversal of iLTP. (c) Representative trace in recordings where we obtained a baseline (black trace) delivered LFS which reduced fEPSP amplitude (red trace) and subsequent TBS, which induced LTP (blue trace). (d) Summary of recordings in which we first delivered LFS then delivered TBS. Numbers on graph correlate with traces in panel (c). Magnitude of pLTP is shaded in blue.

to naive control levels. Therefore, it is likely that there are additional mechanisms that contribute to the LTP impairments in the hippocampus after cerebral ischemia. Regardless, these data suggest that induction of depotentiation to restore physiological plasticity may be a relevant therapy for improving memory function after ischemic brain injury. Future studies should address whether in vivo low-frequency electrical stimulation of the hippocampus, with implanted electrodes or through transmagnetic stimulation, can produce depotentiation and reduce memory deficits in vivo.

In vitro studies have been limited in their ability to record ischemic LTP for only the first hours after ischemia. Here, we are able to show that ischemic LTP is maintained for weeks after injury onset. At 7 and 30 days of postinjury, cell death mechanisms have subsided and recordings are from the surviving hippocampal network. Our ability to depotentiate ischemic LTP and then induce physiological LTP at these delayed time points demonstrates that LTP impairments can be targeted to improve synaptic function, independent of acute neuroprotection. This is an important advance, as acute neuroprotective strategies have failed to improve cognitive outcomes in clinical trials. Cognitive impairments are present in patients that receive therapeutic hypothermia, the only strategy that has given positive results in cardiac arrest victims [39-41]. Therefore, strategies that can provide additional benefit to therapeutic hypothermia have promised to improve neurological function and quality of life for patients. Interestingly, rodent studies have shown that exposure of animals to novel environments can depotentiate 
previously acquired experience-dependent LTP, indicating the potential for novel rehabilitation strategies to reverse iLTP [28]. Future studies should determine whether such a behavioral paradigm could depotentiate ischemic LTP in the intact animal and improve future memory behavior.

In summary, we have demonstrated that in vivo global ischemia produces ischemic LTP which is the result of increased postsynaptic AMPA receptor function. Is iLTP beneficial or detrimental to hippocampal function? Our data demonstrating no change in input-output relations or synaptic density suggest that the hippocampal network is able to compensate for the loss of CA1 neurons after CA/CPR. Ischemic LTP may contribute to this normalization and therefore may have some benefit to the hippocampal network. However, the maintenance of iLTP for weeks after the ischemic insult is detrimental to physiological plasticity and likely worsens memory impairments. Thus, it appears that iLTP may serve as a beneficial compensatory mechanism following brain ischemia that if sustained during the chronic phase is detrimental to long-term recovery. Importantly, we show that this pathological form of plasticity is reversible and thus may be a therapeutic target for cognitive deficits after brain ischemia.

\section{Conflicts of Interest}

The authors declare that they have no conflicts of interest.

\section{Acknowledgments}

This work was supported by AHA BGIA25670032 (Nidia Quillinan) and R01 NS046072 (Nidia Quillinan) and NINDS R01 NS080851 (Paco S. Herson) and R01 NS092645 (Paco S. Herson).

\section{References}

[1] V. Crepel, C. Hammond, P. Chinestra, D. Diabira, and Y. Ben-Ari, "A selective LTP of NMDA receptor-mediated currents induced by anoxia in CA1 hippocampal neurons," Journal of Neurophysiology, vol. 70, no. 5, pp. 2045-2055, 1993.

[2] V. Crepel, C. Hammond, K. Krnjevic, P. Chinestra, and Y. Ben-Ari, "Anoxia-induced LTP of isolated NMDA receptor-mediated synaptic responses," Journal of Neurophysiology, vol. 69, no. 5, pp. 1774-1778, 1993.

[3] K. S. Hsu and C. C. Huang, "Characterization of the anoxiainduced long-term synaptic potentiation in area CA1 of the rat hippocampus," British Journal of Pharmacology, vol. 122, no. 4, pp. 671-681, 1997.

[4] M. Lyubkin, D. M. Durand, and M. A. Haxhiu, "Interaction between tetanus long-term potentiation and hypoxia-induced potentiation in the rat hippocampus," Journal of Neurophysiology, vol. 78, no. 5, pp. 2475-2482, 1997.

[5] N. Maggio, E. Shavit Stein, and M. Segal, "Ischemic LTP: NMDA-dependency and dorso/ventral distribution within the hippocampus," Hippocampus, vol. 25, no. 11, pp. 14651471, 2015.

[6] P. Quintana, S. Alberi, D. Hakkoum, and D. Muller, "Glutamate receptor changes associated with transient anoxia/ hypoglycaemia in hippocampal slice cultures," European Journal of Neuroscience, vol. 23, no. 4, pp. 975-983, 2006.

[7] G. Deng, J. E. Orfila, R. M. Dietz et al., "Autonomous CaMKII activity as a drug target for histological and functional nuroprotection after resuscitation from cardiac arrest," Cell Reports, vol. 18, no. 5, pp. 1109-1117, 2017.

[8] N. Wang, L. Chen, N. Cheng, J. Zhang, T. Tian, and W. Lu, "Active calcium/calmodulin-dependent protein kinase II (CaMKII) regulates NMDA receptor mediated postischemic long-term potentiation (i-LTP) by promoting the interaction between CaMKII and NMDA receptors in ischemia," Neural Plasticity, vol. 2014, Article ID 827161, 10 pages, 2014.

[9] I. Buard, S. J. Coultrap, R. K. Freund et al., "CaMKII "autonomy" is required for initiating but not for maintaining neuronal long-term information storage," The Journal of Neuroscience, vol. 30, no. 24, pp. 8214-8220, 2010.

[10] V. Derkach, A. Barria, and T. R. Soderling, " $\mathrm{Ca}^{2+} /$ calmodulinkinase II enhances channel conductance of $\alpha$-amino-3hydroxy-5-methyl-4-isoxazolepropionate type glutamate receptors," Proceedings of the National Academy of Sciences of the United States of America, vol. 96, no. 6, pp. 3269-3274, 1999.

[11] C. E. Herron, R. A. J. Lester, E. J. Coan, and G. L. Collingridge, "Frequency-dependent involvement of NMDA receptors in the hippocampus: a novel synaptic mechanism," Nature, vol. 322, no. 6076, pp. 265-268, 1986.

[12] R. C. Malenka and R. A. Nicoll, "Long-term potentiation-a decade of progress?," Science, vol. 285, no. 5435, pp. 1870 1874, 1999.

[13] M. Horn and W. Schlote, "Delayed neuronal death and delayed neuronal recovery in the human brain following global ischemia," Acta Neuropathologica, vol. 85, no. 1, pp. 79-87, 1992.

[14] T. Ng, D. I. Graham, J. H. Adams, and I. Ford, "Changes in the hippocampus and the cerebellum resulting from hypoxic insults: frequency and distribution," Acta Neuropathologica, vol. 78, no. 4, pp. 438-443, 1989.

[15] X. Dai, L. Chen, and M. Sokabe, "Neurosteroid estradiol rescues ischemia-induced deficit in the long-term potentiation of rat hippocampal CA1 neurons," Neuropharmacology, vol. 52, no. 4, pp. 1124-1138, 2007.

[16] F. Gillardon, I. Kiprianova, J. Sandkuhler, K. A. Hossmann, and M. Spranger, "Inhibition of caspases prevents cell death of hippocampal CA1 neurons, but not impairment of hippocampal long-term potentiation following global ischemia," Neuroscience, vol. 93, no. 4, pp. 1219-1222, 1999.

[17] I. Kiprianova, J. Sandkühler, S. Schwab, S. Hoyer, and M. Spranger, "Brain-derived neurotrophic factor improves long-term potentiation and cognitive functions after transient forebrain ischemia in the rat," Experimental Neurology, vol. 159, no. 2, pp. 511-519, 1999.

[18] K. Mori, M. Yoshioka, N. Suda et al., "An incomplete cerebral ischemia produced a delayed dysfunction in the rat hippocampal system," Brain Research, vol. 795, no. 1-2, pp. 221-226, 1998.

[19] J. E. Orfila, K. Shimizu, A. K. Garske et al., "Increasing small conductance $\mathrm{Ca}^{2+}$-activated potassium channel activity reverses ischemia-induced impairment of long-term potentiation," European Journal of Neuroscience, vol. 40, no. 8, pp. 3179-3188, 2014. 
[20] R. M. Dietz, G. Deng, J. E. Orfila, X. Hui, R. J. Traystman, and P. S. Herson, "Therapeutic hypothermia protects against ischemia-induced impairment of synaptic plasticity following juvenile cardiac arrest in sex-dependent manner," Neuroscience, vol. 325, pp. 132-141, 2016.

[21] M. P. Hutchens, R. J. Traystman, T. Fujiyoshi, S. Nakayama, and P. S. Herson, "Normothermic cardiac arrest and cardiopulmonary resuscitation: a mouse model of ischemiareperfusion injury," Journal of Visualized Experiments, vol. 54, no. 54, article e3116, 2011.

[22] J. Kofler, K. Hattori, M. Sawada et al., "Histopathological and behavioral characterization of a novel model of cardiac arrest and cardiopulmonary resuscitation in mice," Journal of Neuroscience Methods, vol. 136, no. 1, pp. 33-44, 2004.

[23] N. Quillinan, G. Deng, K. Shimizu et al., "Long-term depression in Purkinje neurons is persistently impaired following cardiac arrest and cardiopulmonary resuscitation in mice," Journal of Cerebral Blood Flow \& Metabolism, vol. 37, no. 8, pp. 3053-3064, 2016.

[24] G. Deng, J. C. Yonchek, N. Quillinan et al., "A novel mouse model of pediatric cardiac arrest and cardiopulmonary resuscitation reveals age-dependent neuronal sensitivities to ischemic injury," Journal of Neuroscience Methods, vol. 222, pp. 34-41, 2014.

[25] Z. I. Bashir and G. L. Collingridge, "An investigation of depotentiation of long-term potentiation in the CA1 region of the hippocampus," Experimental Brain Research, vol. 100, no. 3, pp. 437-443, 1994.

[26] X. Guli, T. Tokay, T. Kirschstein, and R. Kohling, "Status epilepticus enhances depotentiation after fully established LTP in an NMDAR-dependent but GluN2B-independent manner," Neural Plasticity, vol. 2016, Article ID 6592038, 10 pages, 2016.

[27] C. C. Huang, Y. C. Liang, and K. S. Hsu, "Characterization of the mechanism underlying the reversal of long term potentiation by low frequency stimulation at hippocampal CA1 synapses," Journal of Biological Chemistry, vol. 276, no. 51, pp. 48108-48117, 2001.

[28] Y. Qi, N. W. Hu, and M. J. Rowan, "Switching off LTP: mGlu and NMDA receptor-dependent novelty exploration-induced depotentiation in the rat hippocampus," Cerebral Cortex, vol. 23, no. 4, pp. 932-939, 2013.

[29] A. J. Baker, M. H. Zornow, M. R. Grafe et al., "Hypothermia prevents ischemia-induced increases in hippocampal glycine concentrations in rabbits," Stroke, vol. 22, no. 5, pp. 666-673, 1991.

[30] D. Jabaudon, M. Scanziani, B. H. Gahwiler, and U. Gerber, "Acute decrease in net glutamate uptake during energy deprivation," Proceedings of the National Academy of Sciences of the United States of America, vol. 97, no. 10, pp. 5610-5615, 2000.

[31] D. J. Rossi, T. Oshima, and D. Attwell, "Glutamate release in severe brain ischaemia is mainly by reversed uptake," Nature, vol. 403 , no. 6767 , pp. 316-321, 2000.

[32] N. Shimada, R. Graf, G. Rosner, A. Wakayama, C. P. George, and W. D. Heiss, "Ischemic flow threshold for extracellular glutamate increase in cat cortex," Journal of Cerebral Blood Flow \& Metabolism, vol. 9, no. 5, pp. 603-606, 1989.

[33] K. Takata, Y. Takeda, T. Sato, H. Nakatsuka, M. Yokoyama, and K. Morita, "Effects of hypothermia for a short period on histologic outcome and extracellular glutamate concentration during and after cardiac arrest in rats," Critical Care Medicine, vol. 33, no. 6, pp. 1340-1345, 2005.
[34] P. Calabresi, D. Centonze, A. Pisani, L. M. Cupini, and G. Bernardi, "Synaptic plasticity in the ischaemic brain," The Lancet Neurology, vol. 2, no. 10, pp. 622-629, 2003.

[35] M. Di Filippo, A. Tozzi, C. Costa et al., "Plasticity and repair in the post-ischemic brain," Neuropharmacology, vol. 55, no. 3, pp. 353-362, 2008.

[36] K. Kocsis, L. Knapp, L. Gellert et al., “Acetyl-L-carnitine normalizes the impaired long-term potentiation and spine density in a rat model of global ischemia," Neuroscience, vol. 269, pp. 265-272, 2014.

[37] D. Nagy, K. Kocsis, J. Fuzik et al., "Kainate postconditioning restores LTP in ischemic hippocampal CA1: onset-dependent second pathophysiological stress," Neuropharmacology, vol. 61, no. 5-6, pp. 1026-1032, 2011.

[38] G. N. Neigh, E. R. Glasper, J. Kofler et al., "Cardiac arrest with cardiopulmonary resuscitation reduces dendritic spine density in CA1 pyramidal cells and selectively alters acquisition of spatial memory," European Journal of Neuroscience, vol. 20, no. 7, pp. 1865-1872, 2004.

[39] Hypothermia after Cardiac Arrest Study Group, "Mild therapeutic hypothermia to improve the neurologic outcome after cardiac arrest," The New England Journal of Medicine, vol. 346, no. 8, pp. 549-556, 2002.

[40] G. Lilja, N. Nielsen, H. Friberg et al., "Cognitive function in survivors of out-of-hospital cardiac arrest after target temperature management at $33^{\circ} \mathrm{C}$ versus $36^{\circ} \mathrm{C}$," Circulation, vol. 131 , no. 15, pp. 1340-1349, 2015.

[41] M. Tiainen, E. Poutiainen, T. Kovala, O. Takkunen, O. Happola, and R. O. Roine, "Cognitive and neurophysiological outcome of cardiac arrest survivors treated with therapeutic hypothermia," Stroke, vol. 38, no. 8, pp. 2303-2308, 2007. 


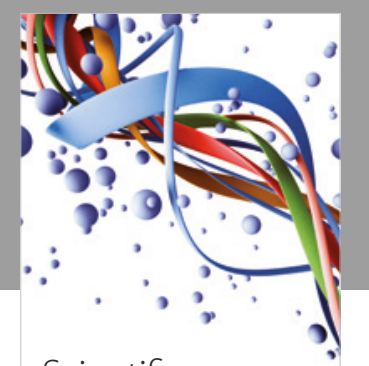

Scientifica
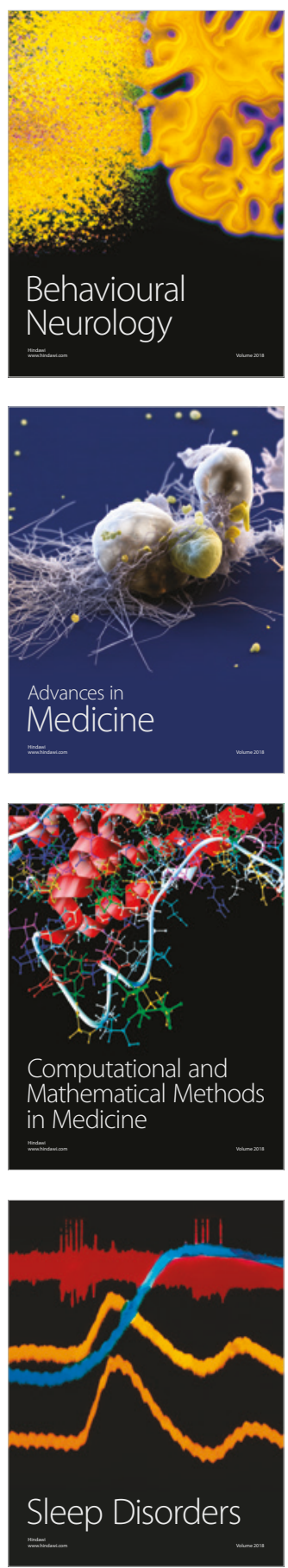

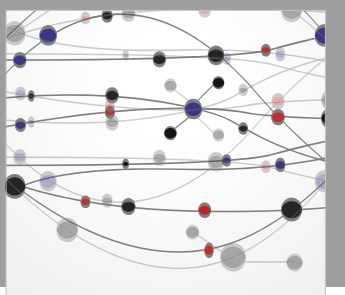

The Scientific World Journal

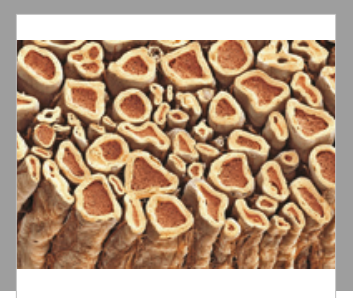

Case Reports in

Neurological Medicine

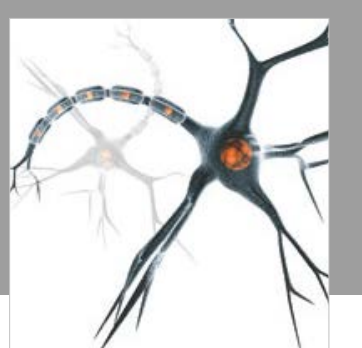

Neural Plasticity

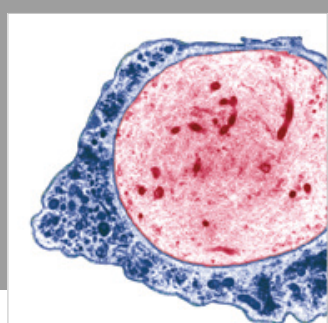

Multiple Sclerosis

International

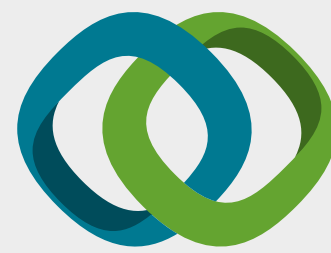

Hindawi

Submit your manuscripts at

www.hindawi.com
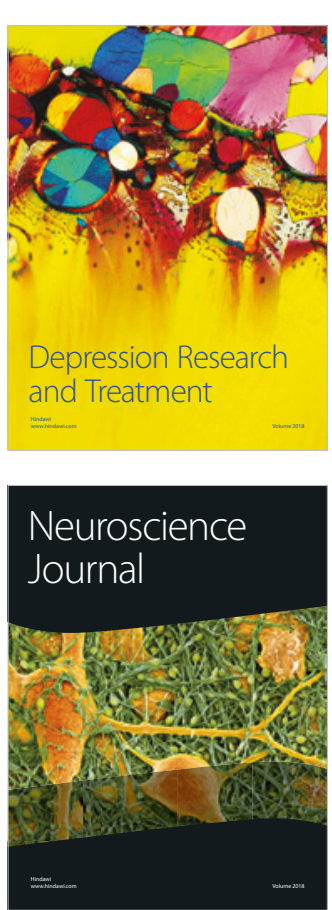



Autism

Research and Treatment
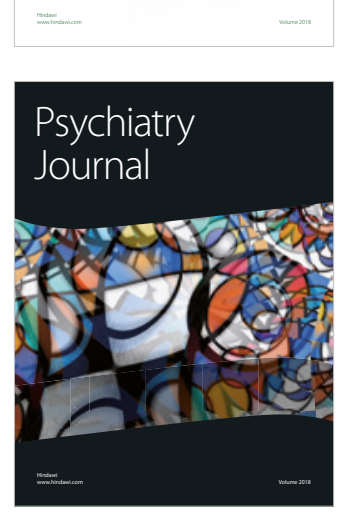

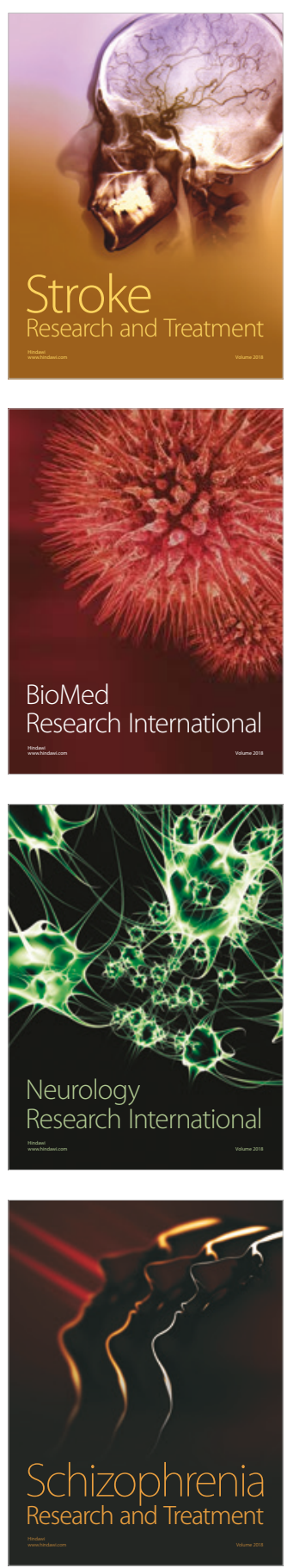